\title{
RESEARCH HIGHLIGHT OPEN Melanoma development: stage-dependent cancer competence of the melanocytic lineage
}

\author{
Manfred Kunz ${ }^{1 凶}$ \\ Signal Transduction and Targeted Therapy (2021)6:433
}

\author{
; https://doi.org/10.1038/s41392-021-00854-3
}

In a recent article in Science, Baggiolini et al. ${ }^{1}$ showed that the transforming ability of the BRAFV600E oncogene for melanoma development depends on the stage of the transformed melanocyte lineage cells. While neural crest cells and melanoblasts are vulnerable to malignant transformation in a zebrafish model, melanocytes only developed small patches of nevus-like cells after BRAFV600E expression.

The tumorigenic potential of DNA mutations depends on the transcriptional and developmental stage and the cellular context in a number of different cancers. ${ }^{2}$ However, this phenomenon has not been studied in detail in malignant melanoma, one of the most aggressive cancers with high metastatic potential. In the mentioned study by Baggiolini et al., ${ }^{1}$ a set of elegant molecular experiments in zebrafish and mice was performed together with additional in vitro experiments. Melanocyte precursors such as neural crest cells and melanoblasts produced aggressive melanomas in p53-/- zebrafish, engineered to express the melanoma oncogene BRAFV600E, while differentiated melanocytes did not (Fig. 1). In these experiments, expression of the BRAFV600E oncogene was put under the control of stage-specific promoters of melanocyte lineage cells, i.e., sox 10 for neural crest cells, mitf for melanoblasts and tyrp 1 for melanocytes. ${ }^{1}$ To further corroborate these findings, human pluripotent stem cells (hPSC) were differentiated into neural crest cells, melanoblasts and mature melanocytes on a triple gene knockout background (3xKO cells). $3 \times$ KO stands for a knockout of tumor suppressor genes RB1, TP53, and p16. BRAFV600E expression was put under the control of a doxycycline-dependent promoter. Subcutaneous injections of either transformed neural crest cells or melanoblasts resulted in tumor formation in immunodeficient NOD scid gamma mice, while injections of transformed melanocytes did not. As shown by RNA-seq analysis, it was further shown that neural crest cells and melanoblasts clustered together with expression profiles of melanoma patient samples of The Cancer Genome Atlas (TCGA). Gene set enrichment analysis showed that pathways enriched in melanoblasts compared to melanocytes were related to chromatin modification, suggesting that epigenetic factors with impact on the chromatin state made these cells competent for melanoma development. Among the top epigenetic-related factors was ATAD2 (ATPAse family AAA domain containing 2). By lentivirusbased induction of ATAD2 expression in 3xKO melanocytes, to establish a progenitor signature, it was subsequently shown by transposase-accessible chromatin analyses (ATAC-seq), that ATAD2 expression opened chromatin at neural-crest related loci. Among transcription factors active at these loci, SOX10 was identified as top candidate. Thus, ATAD2 may specifically support
SOX10 in its binding to target genes. Gene network analysis showed an enrichment of pathways associated with neural crest proliferation and migration. Inactivation of ATAD2 reduced the percentage of neural-crest cell formation in hPSC cells. In line with this, it is well-understood that SOX10 expression is required for melanoma formation in NRAS-mutant mouse melanomas.

TCGA patients with an ATAD2-high signature also showed an enrichment of the SOX10 motif, as well as a strong Myc signature. ${ }^{1}$ In co-immunoprecipitation experiments ATAD2 formed protein complexes with SOX10 and Myc. Co-binding of ATAD2 and SOX10 to ATAD2 target genes was further demonstrated and resulted in the enrichment of the expression of neural-crest related genes. Moreover, genes of the mitogen-activated protein kinase (MAPK) pathway were upregulated in these experiments with $3 \times$ KO cells, including epidermal growth factor receptor (EGFR) and fibroblast growth factor receptor 2 (FGF2), which may have an impact of ERK1/ 2 phosphorylation. This indicates that ATAD2 impacts on neural crest lineage programs and MAPK pathway activation. In a next set of experiments, 3xKO ATAD2 melanocytes were more invasive in invasion chamber assays than $3 \times K O$ cells without ATAD2 induction.

In a final set of experiments, recapitulating the initial experiments, a transgenic zebrafish was generated in which BRAFV600E was overexpressed together with ATAD2 in a p53-/- background to test whether ATAD2 expression might be sufficient for melanoma development in a setting using the tyrp 1 promoter. While tyrp1dependent ATAD2 expressing cells developed melanomas in $10 \%$, with an additional $15 \%$ who developed hyperplastic lesions, none of the ATAD2 non-induced cells developed melanomas. After electroporation of the zebrafish with single-guide RNA targeted against ATAD2, a significant decrease in tumor size was observed. Together, high ATAD2 levels supported the re-expression of a progenitor signature and may thus help mutant $B R A F$ to initiate melanomas.

These findings present in a conclusive way strong experimental evidence that oncogenic pathways must be active at a vulnerable stage of cell development to induce tumor formation, which obviously depends on the cellular context. In a recent study by Belote et al., ${ }^{3}$ transcriptional patterns of melanocytes from different stages of development were analyzed to provide an atlas of human epidermal melanocytes. It was shown that neonatal melanocyte signatures were associated with high treatment resistance and low overall survival in melanoma patients. ${ }^{3}$ Surprisingly, melanocyte stem cell and fetal melanocyte signatures were associated with a better prognosis, but still worse than mature melanocyte signatures. However, survival was largely based on data from treatment with immune-modulatory substances, which were not analyzed in the present study. Moreover,

\footnotetext{
${ }^{1}$ Department of Dermatology, Venereology and Allergology, University Medical Center Leipzig, Philipp-Rosenthal-Str. 23, 04155 Leipzig, Germany
}

Correspondence: Manfred Kunz (manfred.kunz@medizin.uni-leipzig.de)

Received: 14 October 2021 Revised: 8 November 2021 Accepted: 23 November 2021

Published online: 20 December 2021 
Zebrafish p $53-\%$

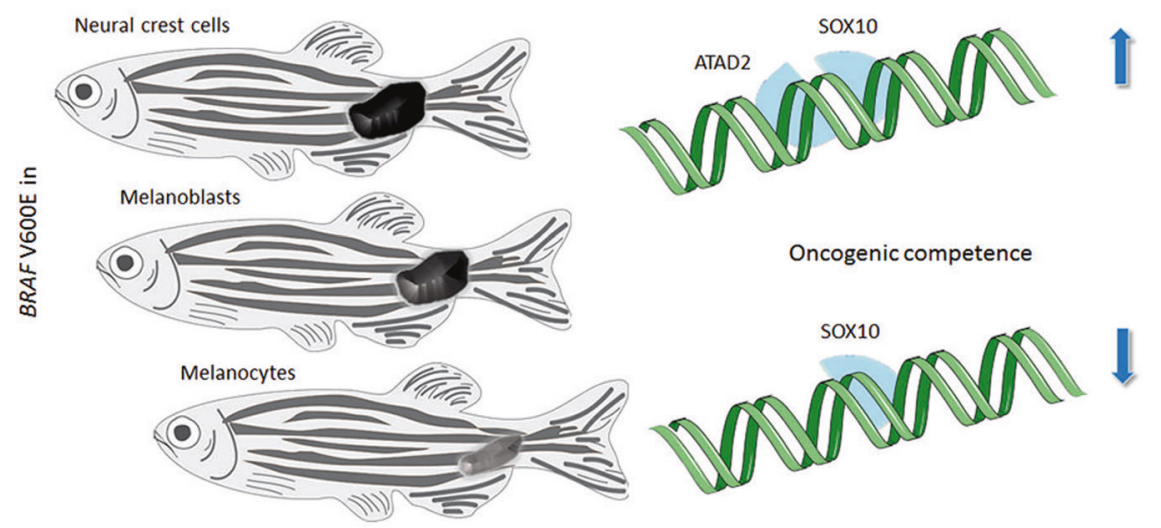

Fig. 1 Oncogenic transformation of $\mathrm{p} 53-/$ - zebrafish results in melanoma formation when the melanoma oncogene BRAFV600E is expressed in neural crest cells or melanoblasts but not when expressed in melanocytes. Epigenetic analyses showed that ATAD2-SOX10 interaction plays a key role in this process

an earlier report on mouse melanocytes showed that melanoblast transcriptomes were indeed associated with melanoma metastasis. ${ }^{4}$ For the moment, Baggiolini et al. ${ }^{1}$ cannot completely explain why established mouse melanoma models where a tyrosinase Cre driver that activates BRAFV600E induces melanomas in the context of PTEN, CDKN2A, and TP53 inactivation. ${ }^{5}$ They argued that the melanoma cells of origin in these experiments have not fully been identified and that the tyrp 1 promoter used in the present study expressed the transgene in a more differentiated type of cells than the tyrosinase (trp) promoter. It is well understood that malignant transformation of melanocytes in vitro has been difficult to obtain with a limited number of oncogenic variants, which supports the notion that differentiated melanocytes might indeed not be the common precursor cells for melanoma. Collectively, the present paper points into a new direction in the understanding of melanoma biology based on an epigenetic re-programming in early stages of melanocyte precursors, which helps to better understand why melanocytes rarely transform into tumors.

\section{ADDITIONAL INFORMATION}

Competing interests: The author has received honoraria from the Speakers Bureau of Roche Pharma and travel support from Novartis Pharma GmbH and Bristol-Myers Squibb $\mathrm{GmbH}$.

\section{REFERENCES}

1. Baggiolini, A. et al. Developmental chromatin programs determine oncogenic competence in melanoma. Science 373, eabc1048 (2021).

2. Haigis, K. M., Cichowski, K. \& Elledge, S. J. Tissue-specificity in cancer: the rule, not the exception. Science 363, 1150-1151 (2019).

3. Belote, R. L. et al. Human melanocyte development and melanoma dedifferentiation at single-cell resolution. Nat. Cell Biol. 23, 1035-1047 (2021).

4. Marie, K. L. et al. Melanoblast transcriptome analysis reveals pathways promoting melanoma metastasis. Nat. Commun. 11, 333 (2020).

5. Pérez-Guijarro, E., Day, C. P., Merlino, G. \& Zaidi, M. R. Genetically engineered mouse models of melanoma. Cancer 123, 2089-2103 (2017).

(c) Open Access This article is licensed under a Creative Commons (c) Attribution 4.0 International License, which permits use, sharing, appropriate credit to the original author(s) and the source, provide a link to the Creative Commons license, and indicate if changes were made. The images or other third party material in this article are included in the article's Creative Commons license, unless indicated otherwise in a credit line to the material. If material is not included in the article's Creative Commons license and your intended use is not permitted by statutory regulation or exceeds the permitted use, you will need to obtain permission directly from the copyright holder. To view a copy of this license, visit http://creativecommons. org/licenses/by/4.0/.

() The Author(s) 2021 